\title{
Cervical cancer screening coverage and its related knowledge in southern Malawi
}

Sibylle Gerstl ${ }^{* *}$, Lawrence Lee ${ }^{1}$, Robin C. Nesbitt ${ }^{1}$, Christopher Mambula ${ }^{2}$, Hartini Sugianto ${ }^{3}$, Twambilire Phiri ${ }^{4}$, James Kachingwe ${ }^{4}$ and Augusto Eduardo Llosa ${ }^{1}$

\begin{abstract}
Background: Cervical cancer (CC) is the fourth most common cancer among women worldwide and Malawi has the world's highest rate of cervical cancer related mortality. Since 2016 the National CC Control Strategy has set a screening coverage target at $80 \%$ of 25-49-year-old women. The Ministry of Health and Médecins Sans Frontières (MSF) set up a CC program in Blantyre City, as a model for urban areas, and Chiradzulu District, as a model for rural areas. This population-based survey aimed to estimate CC screening coverage and to understand why women were or were not screened.

Methods: A population-based survey was conducted in 2019. All resident consenting eligible women aged 25-49years were interviewed $(n=1850)$ at households selected by two-stage cluster sampling. Screening and treatment coverage and facilitators and barriers to screening were calculated stratified by age, weighted for survey design. Chi square and design-based $\mathrm{F}$ tests were used to assess relationship between participant characteristics and screening status.
\end{abstract}

Results: The percentage of women ever screened for CC was highest in Blantyre at 40.2\% (95\% Cl 35.1-45.5), 38.9\% (95\% Cl 32.8-45.4) in Chiradzulu with supported CC screening services, and lowest in Chiradzulu without supported CC screening services at 25.4\% (95\% Cl 19.9-31.8). Among 623 women screened, 49.9\% (95\% Cl 44.0-55.7) reported that recommendation in the health facility was the main reason they were screened and $98.5 \%(95 \% \mathrm{Cl} 96.3-99.4)$ recommended CC screening to others. Among 1227 women not screened, main barriers were lack of time $(26.0 \%, 95 \%$ Cl 21.9-30.6), and lack of motivation (18.3\%, 95\% Cl 14.1-23.3). Overall, 95.6\% (95\% Cl 93.6-97.0) of women reported that they had some knowledge about CC. Knowledge of CC symptoms was low at 34.4\% (95\% Cl 31.0-37.9) and 55.1\% ( $95 \%$ Cl 51.0-59.1) of participants believed themselves to be at risk of CC.

Conclusion: Most of the survey population had heard about CC. Despite this knowledge, fewer than half of eligible women had been screened for CC. Reasons given for not attending screening can be addressed by programs. To significantly reduce mortality due to CC in Malawi requires a comprehensive health strategy that focuses on prevention, screening and treatment.

Keywords: Cervical cancer, Cervical cancer screening, Precancerous lesions, Malawi, Coverage survey, Cross sectional study, Knowledge and attitude, Health behaviours, Barriers, Reproductive health

*Correspondence: sibylle.gerstl@epicentre.msf.org

${ }^{1}$ Epicentre / Médecins Sans Frontières (MSF), Paris, France

Full list of author information is available at the end of the article

\section{Background}

Cervical cancer is the fourth most common cancer among women worldwide for both incidence and mortality [1, 2], and approximately $90 \%$ of cervical cancer deaths occur in low- and middle-income countries [3]. 
Cervical cancer is particularly devastating to individuals, communities and countries as it is a painful cancer that affects women during childbearing and economically active years [4-6].

Cervical cancer is largely a preventable disease; primary prevention and control strategies including human papillomavirus (HPV) vaccination and early detection and treatment of precancerous lesions have contributed to the reduction in disease and mortality burden in many high-income settings with strong health and social systems [2, 7-9]. Cervical cancer mortality has thus become one indicator for functioning and equity of a health care system and has been considered a "a disease of the poor" [3].

Malawi has the highest mortality related to cervical cancer, with 51.5 deaths/100,000/year. This is twice the rate in Eastern Africa (28.6/100,000/year) and seven times the global rate $(7.3 / 100,000 /$ year) [10]. Only surpassed by Swaziland, Malawi has the second highest cervical cancer age-standardized incidence rate in the world (67.9/100,000/year). This compares to a global cervical cancer age-standardized incidence rate of 13.3/100,000/year, and to that of Eastern Africa, the region with the highest cervical cancer incidence rates, with 40.1.7/100,000/year [6, 10, 11].

The high prevalence of Human Immunodeficiency Virus (HIV) among women 15-49 years overall $[12,13]$ in addition to HPV infection, harboured by around $4.8 \%$ of the women in Malawi [14-19], puts Malawian women at higher risk of cervical cancer [20-22].

Health services in Malawi are provided by public, private for profit and private not for profit sectors. Its health system is organized at four levels: community, primary, secondary and tertiary. These different levels are linked to each other through an established referral system. Malawi's health care services, however, experience shortages of essential medical products and technologies and health care financing remains a challenge [23]. In 2016, the Malawi Ministry of Health $(\mathrm{MoH})$ developed the National Cervical Cancer Control Strategy outlining comprehensive interventions to mitigate the burden of cervical cancer [24]. Within this 'Cervical Cancer Control Programme the country adopted the single visit "screen and treat" approach, using visual inspection with acetic acid (VIA) followed by cryotherapy or thermo-coagulation at primary health facility level and with several referral hospitals offering diagnostic and curative cancer services. Cervical cancer screening is mentioned in the 'Essential Health Plan', and public health facilities provide services for free. There is, however, a need for scaling-up VIA screening services in health facilities and increasing resources, such as better infrastructure and human resources, for cervical cancer screening [6]. Current recommendations for VIA screening in Malawi are that women 25-49years are screened once every 3 - 5 years, with yearly screening among HIV positive women. A national target of $80 \%$ screening coverage has been set for women aged 25-49 years being screened with VIA for the first time within the last 12-months [24]. Cervical cancer screening coverage increased from $9 \%$ in 2011 to $26.5 \%$ in 2015 nationwide [25]. According to the Health Sector Joint Annual Review Meeting held by the Directorate of Reproductive Health Services in the MoH on 21 September 2021 , only $34 \%$ of eligible women were screened for cervical cancer between July 2020 and June 2021. Today cervical cancer screening coverage remains well below the target.

Studies on knowledge and awareness of cancer cervical screening carried out in Malawi and surrounding countries showed a consistent mix of screening barriers: little knowledge on symptoms or signs of the disease, lack of information of the screening programme and limited access to screening and treatment [18, 26-34].

Médecins Sans Frontières France (MSF) has been operational in Malawi since 1986 and involved in HIV care since 1997. In 2018, in partnership with the $\mathrm{MoH}$, MSF set up a comprehensive cervical cancer program in Blantyre City, as a model for urban areas, and Chiradzulu District, as a model for rural areas, both situated in the South of the country. By following the "screen and treat" approach the cancer screening component includes health-facility based information sessions and VIA. Immediately after testing, VIA positive patients are offered treatment using thermal ablation in the same health facility by qualified health personnel in Blantyre City and Chiradzulu District. Precancer lesions not treatable by thermal ablation and suspected cancer are referred to a higher level care facility for further diagnosis and treatment. All screen and treat steps are backed up with quality controls. This population-based survey aimed to estimate cervical cancer screening coverage in a representative sample of eligible women in Blantyre City and Chiradzulu District, and to understand why women were or were not screened in order to support the $\mathrm{MoH}$ improve cervical cancer screening uptake in the different areas.

\section{Methods}

\section{Survey design and survey population}

In 2019, a cross-sectional population-based survey was conducted to estimate the prevalence of cervical cancer screening, and to collect reasons for screening among women 25 - 49 years old residing in Blantyre City and Chiradzulu District at the time of the survey. The target sample size was 1815 women in Blantyre City and Chiradzulu District combined. 


\section{Survey area and sampling procedure}

The survey was carried out in three areas, or strata, one in Blantyre City and two in Chiradzulu District. The survey utilized geospatial simple random sampling in Blantyre City, where one GPS coordinate identified one household, all resident consenting eligible women were interviewed. In Chiradzulu District, two-stage cluster sampling was utilized. First, Chiradzulu District was divided into two strata according to proximity to a cervical cancer screening facility supported by MSF; the stratification of the enumeration areas was done by the MSF survey team with the help of the National Statistical Office in Zomba and the health authorities at all levels in Chiradzulu District. Of the 330 EAs (total of 1243 villages) in Chiradzulu District, 161 were classified as having access to MSF-supported cervical cancer services and 169 were classified as without access to MSF-supported cervical cancer services. Within each strata 30 enumeration areas were selected as clusters in the first stage using probability proportional to size. For each cluster, one village was selected in the second stage using systematic random sampling, with 25 households systematically selected and all resident consenting eligible women interviewed.

For simplicity, the names of the three strata will be abbreviated as follows: (1) Blantyre City strata as 'Blantyre', (2) Chiradzulu District with access to MSF-supported cervical cancer services as 'Chiradzulu with supported CC screening services' and, (3) Chiradzulu District without access to MSF-supported cervical cancer services as 'Chiradzulu without CC supported screening services'.

\section{Data collection and questionnaire}

The questionnaire was developed in English and translated into Chichewa, the local language in southern Malawi spoken by the majority of the population. It included questions on knowledge and awareness of cervical cancer, risk factors and prevention, whether the women had ever received cervical cancer screening, and if so details about the screening (including date, location, reasons) and treatment if they received any. Cervical cancer screening was confirmed both by oral history of the interviewee (self-reported) and by the presence of a health passport that contained this information (verified by health passport). The questionnaire was contextadapted based on two models to measure knowledge and awareness of cervical cancer, the 'Cervical Cancer Awareness Measure Toolkit' and the 'Health Belief Model Scale for Cervical Cancer' [35, 36]. It was further tested during the training of the survey team and in a 1-day pilot survey. Data were collected using KoBo Collect (https:// kobo.msf.org) on electronic tablets.

\section{Data analysis}

Data were analysed using Stata version 14.1 (Stata Corp, Texas, USA). Screening and treatment coverage were calculated stratified by age, weighted for survey design. Proportion of women reporting facilitators and barriers to screening were also reported. Chi square and designbased $\mathrm{F}$ tests were used to assess relationship between participant characteristics and screening status in the different geographical areas. Interviews were weighted based on the inverse of probability of selection separately by stratum. In the pooled analysis artificial clusters using the administrative boundaries of the wards were introduced in Blantyre due to the different sampling methodology between the three strata.

\section{Results}

\section{Survey profile}

The survey took place from 24 September to 26 October 2019. A total of 3642 households were visited, and 1824 out of 1885 (96.8\%) households with at least one eligible woman aged 25-49years were interviewed, 45 (2.4\%) refused to participate in the survey. The survey population included a total of 1850 women: 343 (18.5\%) in Blantyre, 755 (40.8\%) in Chiradzulu with supported CC screening services and 752 (40.6\%) in Chiradzulu without supported CC screening services.

\section{Sociodemographic characteristics of survey participants}

The mean age of participants was 34 years overall $($ mean $=33.9$, std. error $=0.2659,95 \%$ CI: $33.3-34.4)$ and did not differ by survey strata, $p=0.13$ (Table 1 ). Literacy was higher in Blantyre where $84.8 \%$ of participants were literate compared to $76.3 \%$ in Chiradzulu without supported CC screening services and 69.4\% in Chiradzulu with supported CC screening services, $p<0.001$. Working outside of the home was more common in Blantyre than in either strata in Chiradzulu, and the proportion of women reporting the highest category of average monthly household income was higher in Blantyre at $43.7 \%$ compared to 12.2 and $8.0 \%$ in Chiradzulu with and without supported CC screening services respectively, $\mathrm{p}<0.001$. The proportion of HIV positive participants on anti-retroviral therapy (ART) was lower in Blantyre at $14.9 \%$ than in Chiradzulu with supported CC screening services at $25.6 \%$ and without supported CC screening services at $22.6 \%$, $p=0.006$.

\section{Cervical cancer screening coverage}

The percentage of women aged 25-49years ever screened for cervical cancer (either verified by health passport or self-reported) differed by stratum, and was highest in Blantyre at 40.2\% (138/343, 95\% CI 


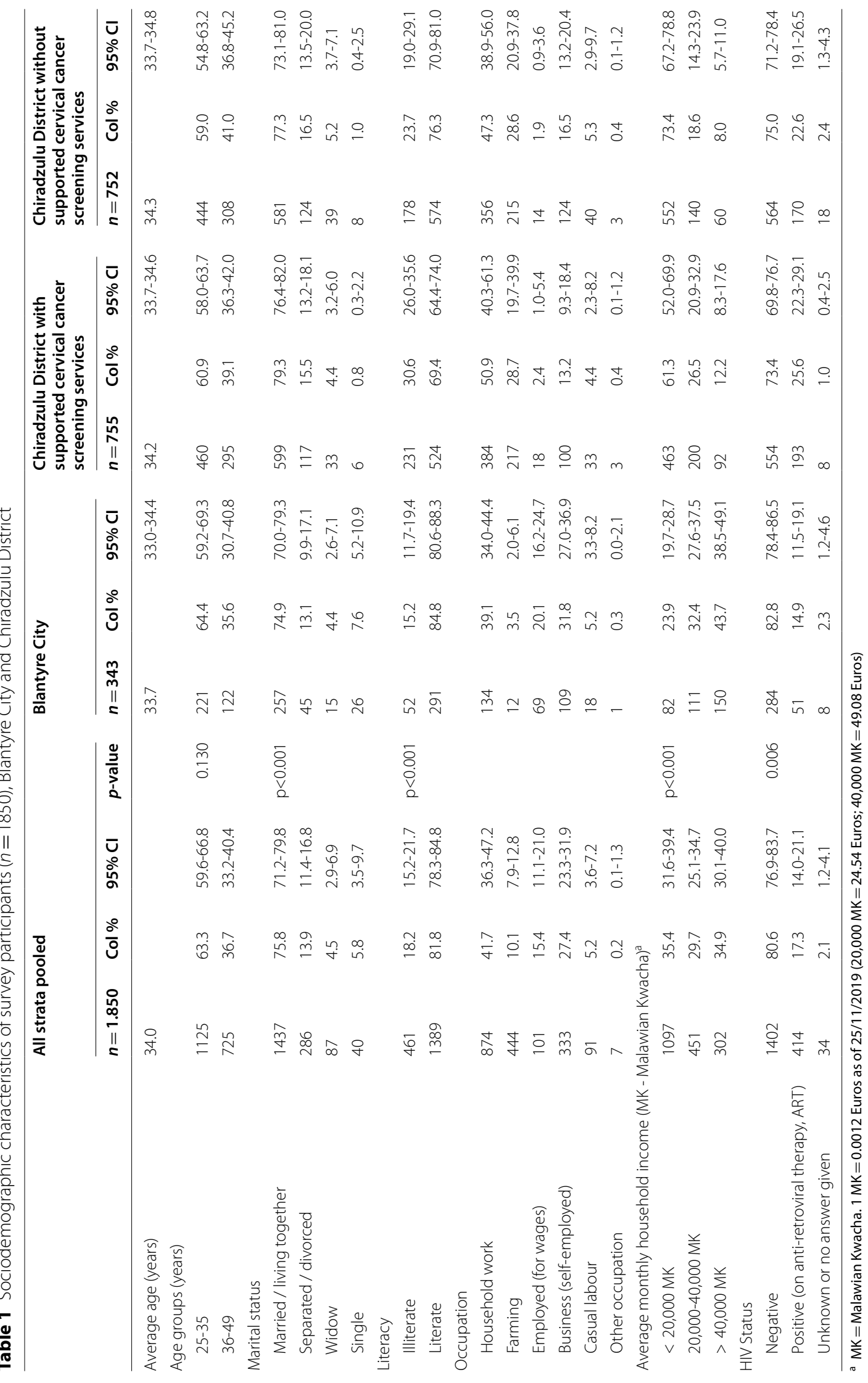


35.1-45.5), 38.9\% (294/755, 95\% CI 32.8-45.4) in Chiradzulu with supported $\mathrm{CC}$ screening services, and lowest in Chiradzulu without supported CC screening services at 25.4\% (191/752, 95\% CI 19.9-31.8) (Fig. 1). Screening coverage of women aged 25-49 years was statistically significantly greater in Chiradzulu with supported CC screening services compared to Chiradzulu without supported CC screening services $(p=0.003)$. The estimated screening coverage considering only those verified in the health passport, was $23.6 \%(81 / 343$, 95\% CI 19.4-28.4) in Blantyre; 31.7\% (239/755, 95\% CI 26.1-37.8) in Chiradzulu with access to supported CC services, and $19.0 \%$ (143/752, 95\% CI 14.1-25.2) in Chiradzulu without access to supported CC services.

The percentage of survey participants screened multiple times for cervical cancer was 9.9\% (34/343, 95\% CI 7.2-13.6) in Blantyre; 6.9\% (52/755, 95\% CI 4.7-10.0) in Chiradzulu with access to supported CC screening services; and 3.2\% (24/752, 95\% CI 2.1-4.8) in Chiradzulu without access to supported $\mathrm{CC}$ screening services.

\section{Results of cervical cancer screening}

Among the survey participants who were screened, 4 (0.2, 95\% CI 0.1-0.6) were VIA positive (3 verified in health passport and 1 self-reported) and 1 (0.1, 95\% CI $0.01-0.4$ ) was identified as suspect cancer, verified in the health passport.

Three of the four VIA positive survey participants and the suspect cancer case received treatment. The VIA positive participant that did not receive treatment reported that treatment was not necessary. Three out of the four VIA positive survey participants and the suspect cancer case were HIV positive on ART.

\section{Characteristics associated with cervical cancer screening among women screened in the different geographical areas}

Considering characteristics associated with screening by survey site, older age and literacy were consistently associated with being screened for cervical cancer in all three geographical areas, whereas other characteristics differed. Education was associated with screening

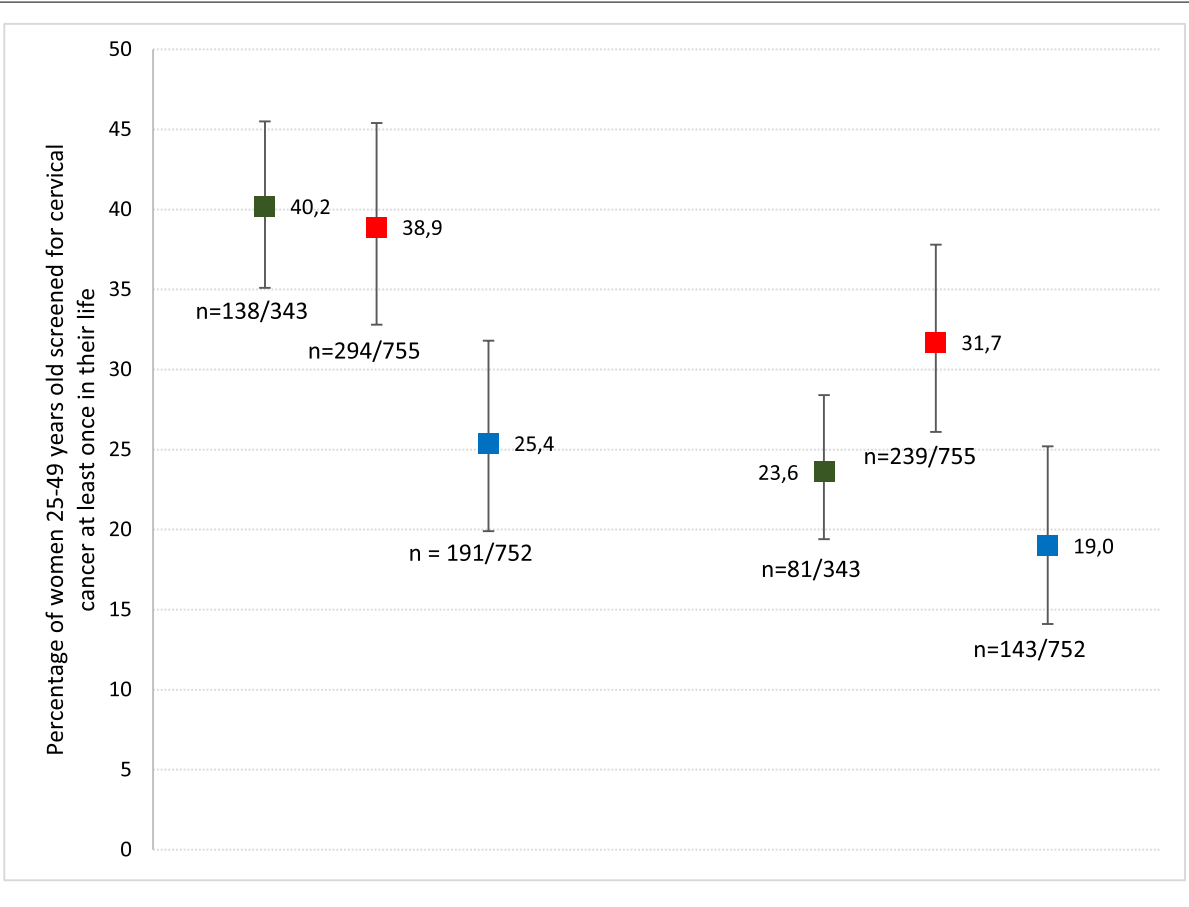

Verified by health passport or self-reported

Verified by health passport only

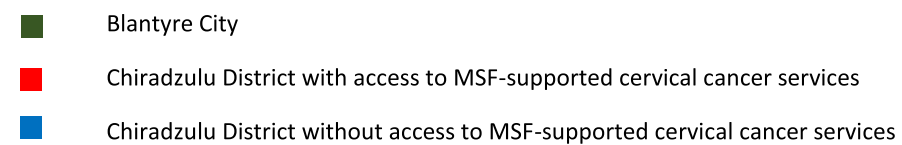

Fig. 1 Estimated percentage of women 25-49years who have ever been screened for cervical cancer by stratum, Blantyre City and Chiradzulu District, Southern Malawi, 2019 
in Blantyre only, and income level was associated with screening in Blantyre and Chiradzulu without access to supported CC screening services, but not Chiradzulu with access to supported CC screening services. HIV status significantly was associated with screening in both Chiradzulu sites. In Blantyre, where overall proportion of HIV positive participants was lower, there was no association between screening and HIV status (Table 2).

\section{Reasons for cervical cancer screening}

Among 623 women screened overall, 334 (49.9, 95\% CI 44.0-55.7) reported that recommendation in the health facility was the main reason they were screened. This proportion was $48.6 \%$ (95\% CI 40.2-57.0) in Blantyre, $60.5 \%$ (95\% CI 52.0-68.5) in Chiradzulu with supported CC screening services, and $46.6 \%$ (95\% CI 35.4-58.2) in Chiradzulu without supported $\mathrm{CC}$ screening services. Other common reasons included recommended by screening campaign (12.4\% overall, 95\% CI 9.0-16.7), self-volition (10.8, 95\% CI 6.5-17.5) and recommended by family and friends (10.2, 95\% CI7.1-14.4). Overall, 98.5\% (95\% CI 96.3-99.4) of women who were screened reported that they would recommend cervical cancer screening to others; this was consistent across all three strata (Table 3).

\section{Reasons for not screening}

Among 1227 women not screened overall, the main two reasons for not being screened were lack of time for screening (26.0, 95\% CI 21.9-30.6), and lack of motivation to go for screening (18.3, 95\% CI 14.1-23.3). Reasons for not being screened differed according to strata. In Blantyre, 30.2\% (95\% CI 24.3-36.9) of women reported lack of time for screening as the main reasons they were not screened and $20.1 \%$ (95\% CI 15.0-26.1) reported lack of motivation. In Chiradzulu without supported CC screening services the most common reasons were that the location was not convenient (21.9, 95\% CI 15.6-29.9), followed by lack of information (18.5, 95\% CI 14.6-23.3), whereas lack of time for screening was less important, reported by $13.4 \%$ (95\% CI 9.0-19.3). In Chiradzulu with supported CC screening services, lack of information 19.1\% (95\% CI 13.2-26.8), lack of time for screening (18.9, 95\% CI 14.2-24.7), and lack of motivation $18.4 \%$ (95\% CI 13.3-25.0) were the top reasons reported by similar proportions of women (Table 4).

Fear of screening was not a predominant concern, overall 149 (13.7, 95\% CI 11.1-16.9) women who were not screened reported fear as the main reason for not screening (Table 4). Furthermore, only 49 (4.9, 95\% CI 3.0-7.8) of women not screened said they were not willing to be screened by a man. Overall $62.2 \%$ (95\% CI 57.3-66.9) of women who were not screened showed a willingness to pay for this service. This number was lower in both strata in Chiradzulu district than Blantyre (Table 4).

\section{Knowledge, awareness and beliefs about cervical cancer and cervical cancer screening}

Overall, 95.6\% (95\% CI 93.6-97.0) of women reported that they had knowledge about cervical cancer, this proportion was similar among participants who had not been screened for cervical cancer themselves at $94.0 \%$ (95\% CI 91.4-95.8, $p=0.069$ ). Fewer women reported knowledge of screening for cervical cancer, and this did significantly differ between women who were screened themselves and those who were not screened, 74.8\% (95\% CI 70.3-78.9, $p<0.001$ ) overall and $64.6 \%$ (95\% CI 58.1$70.5, \mathrm{p}<0.001)$ among women who were not screened themselves. Knowledge of cervical cancer symptoms was significantly low at overall $34.4 \%$ (95\% CI 31.0-37.9, $\mathrm{p}<0.001)$ and $24.6 \%(95 \%$ CI 21.8-27.6, $\mathrm{p}<0.001)$ among women who had not been screened for cervical cancer themselves (Table 5).

Overall, 55.1\% (95\% CI 51.0-59.1) of participants believed themselves to be at risk of cervical cancer, this was highest among those never screened themselves (56.3, 95\% CI 51.6-60.9, $p=0.006$ ). Additionally, 20.3\% (95\% CI 16.1-25.3) of the women not screened did not know if they were at risk of cervical cancer. In contrast, a high proportion of women reported awareness that cervical cancer can be prevented (68.1, 95\% CI 64.2-71.8) and cured (73.3, 95\% CI 70.1-76.2), and this was higher among women who were screened compared to women not screened, $p=0.001$ for both indicators. There was a similar and relatively high level of knowledge on prevention methods (having regular medical check-ups, being faithful to their partner, delaying sexual debut), and HIV as a risk factor among women who were and were not screened. However, $36.4 \%$ of women believed in witchcraft as cause for cervical cancer or were not sure about it (Table 5).

\section{Discussion}

Overall, despite relatively high knowledge of cervical cancer, cervical cancer screening coverage in Blantyre City and Chiradzulu District remained well below the national goal of $80 \%$. Coverage was highest in Blantyre with almost every second eligible women screened, compared to slightly more than every third women screened in Chiradzulu with supported CC screening services and only every fourth women in Chiradzulu without supported CC screening services. Few women in our survey were screened more than once. This suggests that screening programs continue to reach women with cervical cancer screening for the first time, and that there remains 


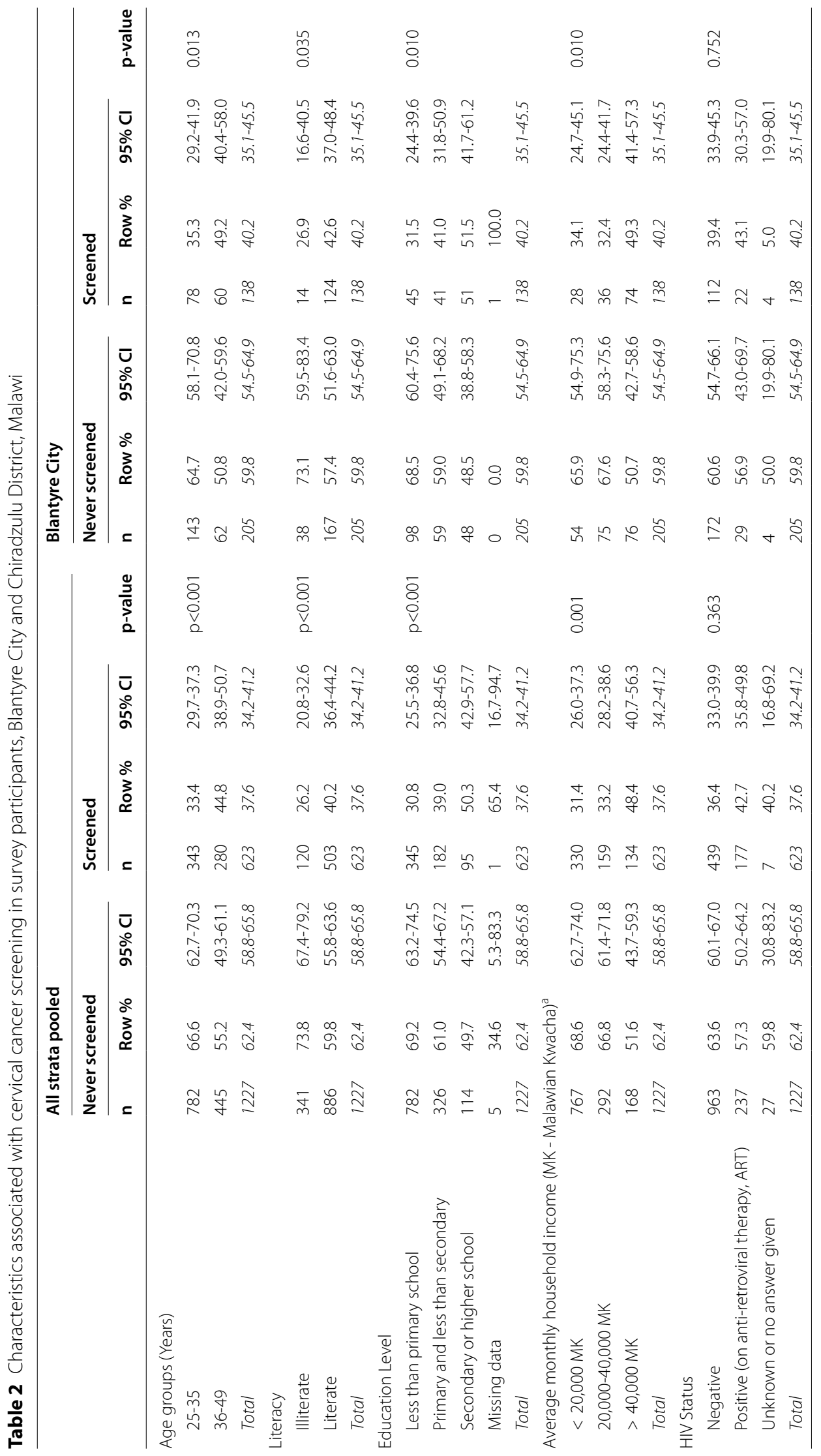




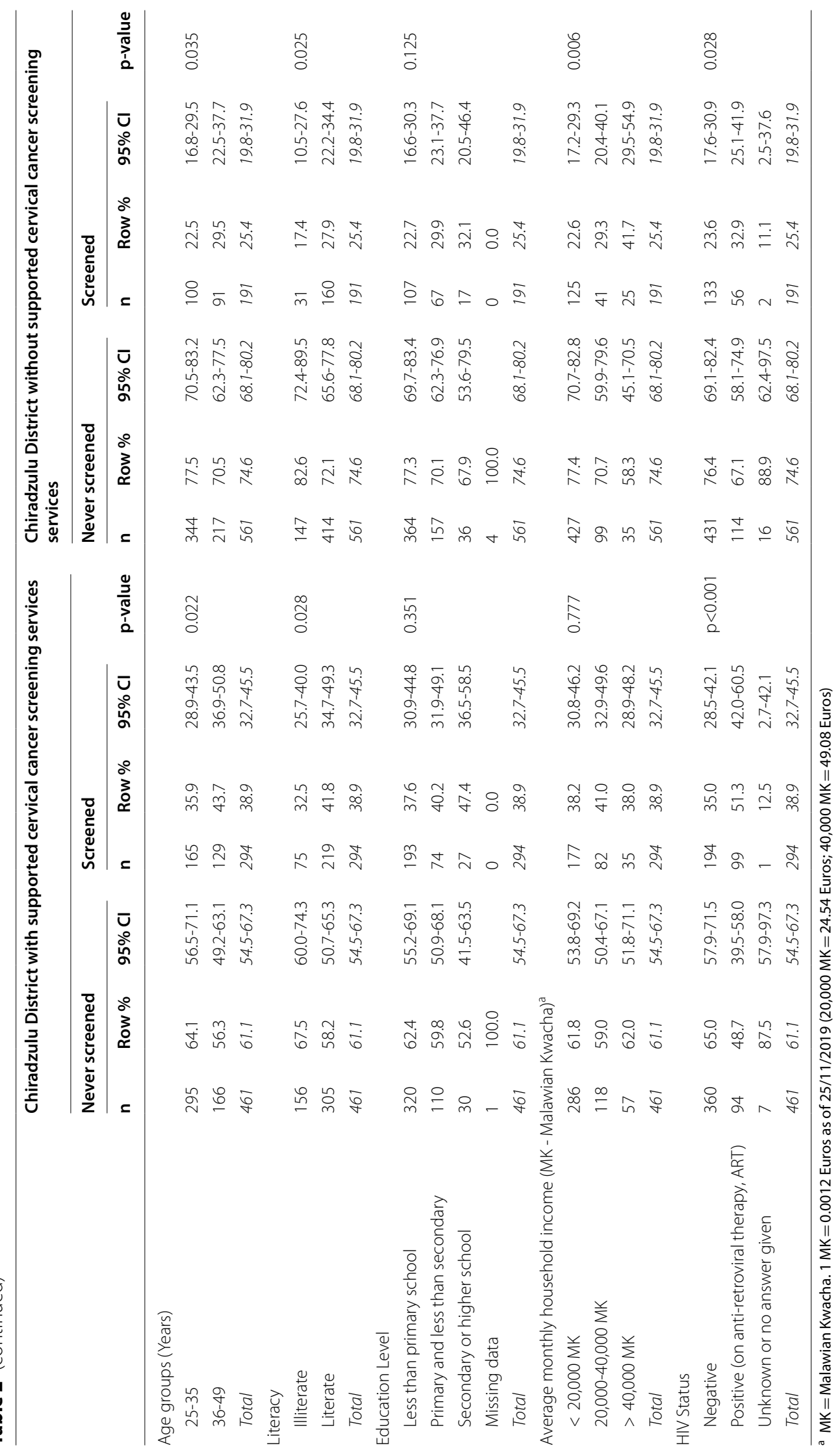


Table 3 Reasons for cervical cancer screening among women screened ( $n=623)$, Blantyre City and Chiradzulu District, Malawi

\begin{tabular}{|c|c|c|c|c|c|c|c|c|c|c|c|c|}
\hline & \multicolumn{3}{|c|}{ All strata pooled } & \multicolumn{3}{|c|}{ Blantyre City } & \multicolumn{3}{|c|}{$\begin{array}{l}\text { Chiradzulu District } \\
\text { with supported } \\
\text { cervical cancer } \\
\text { screening services }\end{array}$} & \multicolumn{3}{|c|}{$\begin{array}{l}\text { Chiradzulu District } \\
\text { without supported } \\
\text { cervical cancer } \\
\text { screening services }\end{array}$} \\
\hline & $\mathbf{n}$ & Col. \% & $95 \% \mathrm{Cl}$ & $\mathbf{n}$ & Col. \% & $95 \% \mathrm{Cl}$ & $\mathbf{n}$ & Col. \% & $95 \% \mathrm{Cl}$ & $\mathbf{n}$ & Col. \% & $95 \% \mathrm{Cl}$ \\
\hline \multicolumn{13}{|l|}{ Main reason for cervical cancer screening } \\
\hline Recommended in health facility & 334 & 49.9 & $44.0-55.7$ & 67 & 48.6 & $40.2-57.0$ & 178 & 60.5 & $52.0-68.5$ & 89 & 46.6 & $35.4-58.2$ \\
\hline Self-volition & 77 & 10.8 & $6.5-17.5$ & 14 & 10.1 & $6.1-16.5$ & 29 & 9.9 & $5.9-16.0$ & 34 & 17.8 & $11.8-26.0$ \\
\hline Recommended by family / friends & 64 & 10.2 & 7.1-14.4 & 14 & 10.1 & $6.1-16.5$ & 31 & 10.5 & 7.3-15.0 & 19 & 9.9 & $6.1-15.9$ \\
\hline Recommended by screening campaigns & 54 & 12.4 & $9.0-16.7$ & 19 & 13.8 & 8.9-20.7 & 17 & 5.8 & 3.6-9.1 & 18 & 9.4 & 6.3-13.9 \\
\hline Recommended by media & 37 & 8.4 & $5.6-12.5$ & 13 & 9.4 & $5.5-15.6$ & 17 & 5.8 & 3.4-9.8 & 7 & 3.7 & $1.7-7.8$ \\
\hline Other reason & 54 & 8.2 & $4.5-14.4$ & 11 & 8.0 & 4.4-13.9 & 19 & 6.5 & $3.7-11.0$ & 24 & 12.6 & 7.9-19.5 \\
\hline No reason given & 3 & 0.1 & $0.0-0.4$ & 0 & 0 & & 3 & 1.0 & $0.3-3.2$ & 0 & 0 & \\
\hline Total & 623 & 1 & & 138 & 100 & & 294 & 100 & & 191 & 100 & \\
\hline \multicolumn{13}{|c|}{ Would recommend cervical cancer screening to others } \\
\hline No & 10 & 1.5 & $0.6-3.7$ & 2 & 1.4 & $0.4-5.7$ & 8 & 2.7 & $1.2-6.3$ & 0 & 0 & \\
\hline Yes & 613 & 98.5 & $96.3-99.4$ & 136 & 98.6 & $94.3-99.6$ & 286 & 97.3 & $93.7-98.8$ & 191 & 100 & \\
\hline Total & 623 & 100 & & 138 & 100 & & 294 & 100 & & 191 & 100 & \\
\hline
\end{tabular}

Table 4 Reasons for not screening among women not screened ( $n=1227)$, Blantyre City and Chiradzulu District, Malawi

\begin{tabular}{|c|c|c|c|c|c|c|c|c|c|c|c|c|}
\hline & \multicolumn{3}{|c|}{ All strata pooled } & \multicolumn{3}{|c|}{ Blantyre City } & \multicolumn{3}{|c|}{$\begin{array}{l}\text { Chiradzulu District } \\
\text { with supported } \\
\text { cervical cancer } \\
\text { screening services }\end{array}$} & \multicolumn{3}{|c|}{$\begin{array}{l}\text { Chiradzulu District } \\
\text { without supported } \\
\text { cervical cancer } \\
\text { screening services }\end{array}$} \\
\hline & $\mathrm{n}$ & Col. \% & $95 \% \mathrm{Cl}$ & $\mathbf{n}$ & Col. \% & $95 \% \mathrm{Cl}$ & $\mathbf{n}$ & Col. \% & $95 \% \mathrm{Cl}$ & $\mathrm{n}$ & Col. \% & $95 \% \mathrm{Cl}$ \\
\hline \multicolumn{13}{|l|}{ Main reason not screened } \\
\hline Lack of time for screening & 224 & 26.0 & 21.9-30.6 & 62 & 30.2 & 24.3-36.9 & 87 & 18.9 & $14.2-24.7$ & 75 & 13.4 & $9.0-19.3$ \\
\hline Lack of information about screening & 216 & 13.7 & 10.3-18.1 & 24 & 11.7 & 7.9-16.9 & 88 & 19.1 & $13.2-26.8$ & 104 & 18.5 & $14.6-23.3$ \\
\hline Screening location not convenient & 197 & 10.4 & 8.0-13.4 & 15 & 7.3 & 4.4-11.8 & 59 & 12.8 & 8.4-19.0 & 123 & 21.9 & 15.6-29.9 \\
\hline Lack of motivation to go for screening & 187 & 18.3 & $14.1-23.3$ & 41 & 20.1 & $15.0-26.1$ & 85 & 18.4 & 13.3-25.0 & 61 & 10.9 & $7.2-16.0$ \\
\hline Fear of screening & 149 & 13.7 & $11.1-16.9$ & 30 & 14.6 & $10.4-20.2$ & 61 & 13.2 & $10.2-16.9$ & 58 & 10.3 & 7.4-14.3 \\
\hline Lack of screening capacity at health facility & 58 & 3.9 & $2.5-6.0$ & 7 & 3.4 & $1.6-7.0$ & 21 & 4.6 & $2.6-7.9$ & 30 & 5.3 & $2.8-9.9$ \\
\hline Other reason & 41 & 3.4 & $2.0-5.8$ & 7 & 3.4 & $1.6-7.0$ & 8 & 1.7 & $0.8-3.7$ & 26 & 4.6 & $3.1-6.9$ \\
\hline No reason given & 155 & 10.5 & 5.9-17.9 & 19 & 9.3 & $6.0-14.1$ & 52 & 11.3 & $7.8-16.0$ & 84 & 15.0 & $9.5-22.8$ \\
\hline Total & 1227 & 100 & & 205 & 100 & & 461 & 100 & & 561 & 100 & \\
\hline \multicolumn{13}{|l|}{ Would be screened by men } \\
\hline No & 49 & 4.9 & $3.0-7.8$ & 11 & 5.3 & $3.0-9.5$ & 17 & 3.7 & $2.1-6.5$ & 21 & 3.7 & $2.4-5.7$ \\
\hline Yes & 1171 & 94.3 & $91.3-96.3$ & 192 & 93.7 & $89.3-96.3$ & 441 & 95.7 & $93.0-97.3$ & 538 & 95.9 & $93.8-97.3$ \\
\hline Do not know & 7 & 0.8 & $0.2-2.8$ & 2 & 1.0 & $0.2-3.9$ & 3 & 0.6 & $0.2-2.0$ & 2 & 0.4 & $0.1-1.4$ \\
\hline Total & 1227 & 100 & & 205 & 100 & & 461 & 100 & & 561 & 100 & \\
\hline \multicolumn{13}{|l|}{ Would pay for screening } \\
\hline No & 548 & 37.8 & $33.1-42.7$ & 70 & 34.1 & $27.9-41.0$ & 217 & 47.1 & $41.4-52.8$ & 261 & 46.5 & $40.1-53.1$ \\
\hline Yes & 679 & 62.2 & $57.3-66.9$ & 135 & 65.9 & $59.0-72.1$ & 244 & 52.9 & $47.2-58.6$ & 300 & 53.5 & $46.9-59.9$ \\
\hline Total & 1227 & 100 & & 205 & 100 & & 461 & 100 & & 561 & 100 & \\
\hline
\end{tabular}


Table 5 Knowledge, awareness and beliefs about cervical cancer and cervical cancer screening in the survey participants

\begin{tabular}{|c|c|c|c|c|c|c|c|c|c|c|c|c|c|}
\hline & \multicolumn{3}{|c|}{$\begin{array}{l}\text { Screened by health } \\
\text { centers with supported } \\
\text { cervical cancer services } \\
\text { (health passport or self- } \\
\text { report) }\end{array}$} & \multicolumn{3}{|c|}{$\begin{array}{l}\text { Screened by health } \\
\text { centers without } \\
\text { supported cervical cancer } \\
\text { services (health passport } \\
\text { or self-report) }\end{array}$} & \multicolumn{3}{|c|}{$\begin{array}{l}\text { Never screened for cervical } \\
\text { cancer }\end{array}$} & \multirow[t]{2}{*}{ Total } & \multirow[b]{2}{*}{ Col. \% } & \multirow[b]{2}{*}{$95 \% \mathrm{Cl}$} & \multirow[b]{2}{*}{$p$-value } \\
\hline & $\mathbf{n}$ & Col. \% & $95 \% \mathrm{Cl}$ & $\mathbf{n}$ & Col. \% & $95 \% \mathrm{Cl}$ & $\mathbf{n}$ & Col. \% & $95 \% \mathrm{Cl}$ & & & & \\
\hline \multicolumn{14}{|c|}{ Knowledge of cervical cancer } \\
\hline No & 3 & 2.1 & $0.4-11.7$ & 3 & 1.7 & $0.5-6.0$ & 111 & 6.0 & $4.2-8.6$ & 117 & 4.4 & $3.0-6.4$ & 0.069 \\
\hline Yes & 298 & 97.9 & 88.3-99.6 & 319 & 98.3 & $94.0-99.5$ & 1116 & 94.0 & $91.4-95.8$ & 1733 & 95.6 & $93.6-97.0$ & \\
\hline Total & 301 & 100 & & 322 & 100 & & 1227 & 100 & & 1850 & 100 & & \\
\hline \multicolumn{14}{|c|}{ Knowledge of cervical cancer screening } \\
\hline No & 21 & 6.4 & $2.6-15.0$ & 37 & 9.3 & $6.1-14.0$ & 448 & 34.4 & $28.6-40.7$ & 506 & 24.5 & $20.5-29.0$ & $p<0.001$ \\
\hline Yes & 279 & 93.5 & 84.9-97.3 & 284 & 90.6 & $86.0-93.8$ & 765 & 64.6 & $58.1-70.5$ & 1328 & 74.8 & 70.3-78.9 & \\
\hline Do not know & 1 & 0.1 & $0.0-1.0$ & 1 & 0.1 & $0.0-0.5$ & 14 & 1.1 & $0.4-2.7$ & 16 & 0.7 & $0.3-1.7$ & \\
\hline Total & 301 & 100 & & 322 & 100 & & 1227 & 100 & & 1850 & 100 & & \\
\hline \multicolumn{14}{|c|}{ Knowledge of cervical cancer symptoms } \\
\hline No & 151 & 50.0 & $41.5-58.5$ & 156 & 49.6 & $41.3-58.0$ & 898 & 75.4 & 72.4-78.2 & 1205 & 65.6 & $62.1-69.0$ & $p<0.001$ \\
\hline Yes & 150 & 50.0 & $41.5-58.5$ & 166 & 50.4 & $42.0-58.7$ & 329 & 24.6 & $21.8-27.6$ & 645 & 34.4 & $31.0-37.9$ & \\
\hline Total & 301 & 100 & & 322 & 100 & & 1227 & 100 & & 1850 & 100 & & \\
\hline \multicolumn{14}{|c|}{ Awareness of being at risk for cervical cancer themselves } \\
\hline No & 118 & 43.6 & $33.3-54.5$ & 82 & 28.8 & $22.0-36.8$ & 217 & 23.4 & 19.7-27.4 & 417 & 27.2 & $23.3-31.4$ & 0.006 \\
\hline Yes & 167 & 50.8 & $40.0-61.5$ & 199 & 54.1 & $45.8-62.2$ & 809 & 56.3 & $51.6-60.9$ & 1175 & 55.1 & $51.0-59.1$ & \\
\hline Do not know & 16 & 5.6 & $2.6-11.6$ & 41 & 17.1 & $10.1-27.3$ & 201 & 20.3 & $16.1-25.3$ & 258 & 17.7 & $14.4-21.6$ & \\
\hline Total & 301 & 100 & & 322 & 100 & & 1227 & 100 & & 1850 & 100 & & \\
\hline \multicolumn{14}{|c|}{ Awareness of possibility to prevent cervical cancer } \\
\hline No & 61 & 15.3 & 11.0-20.9 & 52 & 11.0 & 7.5-15.9 & 292 & 20.6 & $16.5-25.5$ & 405 & 17.5 & $14.5-20.9$ & 0.001 \\
\hline Yes & 212 & 75.7 & $68.2-81.8$ & 242 & 79.6 & 74.6-83.8 & 722 & 61.9 & $55.7-67.7$ & 1176 & 68.1 & $64.2-71.8$ & \\
\hline Do not know & 28 & 9.0 & $5.0-15.9$ & 28 & 9.4 & $5.6-15.5$ & 213 & 17.5 & $13.6-22.3$ & 269 & 14.4 & $11.8-17.5$ & \\
\hline Total & 301 & 100 & & 322 & 100 & & 1227 & 100 & & 1850 & 100 & & \\
\hline \multicolumn{14}{|c|}{ Awareness of possibility to cure cervical cancer } \\
\hline No & 50 & 13.8 & $7.8-23.0$ & 60 & 13.1 & $8.3-20.0$ & 277 & 21.2 & $17.7-25.2$ & 387 & 18.2 & $15.7-21.0$ & 0.001 \\
\hline Yes & 240 & 84.7 & $76.2-90.5$ & 244 & 81.9 & 74.0-87.9 & 798 & 67.5 & $63.0-71.6$ & 1282 & 73.3 & $70.1-76.2$ & \\
\hline Do not know & 11 & 1.5 & $0.8-3.1$ & 18 & 5.0 & $2.2-10.7$ & 152 & 11.3 & $9.2-13.9$ & 181 & 8.5 & 7.1-10.3 & \\
\hline Total & 301 & 100 & & 322 & 100 & & 1227 & 100 & & 1850 & 100 & & \\
\hline \multicolumn{14}{|c|}{ Knowledge on prevention methods, such as: } \\
\hline \multicolumn{14}{|c|}{ - Medical check-up, VIA screening, HPV vaccination } \\
\hline True & 287 & 94.6 & 86.9-97.9 & 300 & 91.7 & $84.8-95.7$ & 1119 & 92.4 & 88.9-94.9 & 1706 & 92.5 & $89.6-94.7$ & 0.602 \\
\hline False & 10 & 4.8 & $1.7-12.9$ & 16 & 4.8 & $2.2-10.2$ & 69 & 5.2 & $3.4-7.8$ & 95 & 5.0 & $3.4-7.3$ & \\
\hline Do not know & 4 & 0.6 & $0.2-1.6$ & 6 & 3.4 & $1.6-7.3$ & 39 & 2.4 & $1.4-4.2$ & 49 & 2.5 & $1.6-3.8$ & \\
\hline Total & 301 & 100 & & 322 & 100 & & 1227 & 100 & & 1850 & 100 & & \\
\hline \multicolumn{14}{|c|}{ - Being faithful to a sexual partner } \\
\hline True & 270 & 85.5 & 74.6-92.2 & 290 & 89.6 & $83.7-93.5$ & 1033 & 87.5 & $84.1-90.2$ & 1593 & 87.8 & 84.3-90.6 & 0.332 \\
\hline False & 25 & 12.0 & $6.0-22.5$ & 23 & 5.3 & $2.8-9.9$ & 143 & 8.5 & $6.6-10.9$ & 191 & 8.1 & $6.1-10.6$ & \\
\hline Do not know & 6 & 2.5 & $0.6-10.6$ & 9 & 5.1 & $2.4-10.8$ & 51 & 4.0 & $2.6-6.2$ & 66 & 4.1 & $2.7-6.3$ & \\
\hline Total & 301 & 100 & & 322 & 100 & & 1227 & 100 & & 1850 & 100 & & \\
\hline \multicolumn{14}{|c|}{ - Delaying sexual debut } \\
\hline True & 173 & 59.9 & $51.3-67.9$ & 206 & 63.6 & $57.4-69.3$ & 663 & 55.7 & $51.2-60.1$ & 1042 & 58.3 & $54.8-61.6$ & 0.134 \\
\hline False & 84 & 28.8 & $18.2-42.4$ & 66 & 18.7 & $14.4-23.9$ & 352 & 27.1 & $22.6-32.2$ & 502 & 25.1 & $21.7-28.8$ & \\
\hline Do not know & 44 & 11.3 & $5.6-21.4$ & 50 & 17.7 & $12.9-23.8$ & 212 & 17.2 & $13.9-21.1$ & 306 & 16.6 & $13.8-19.8$ & \\
\hline Total & 301 & 100 & & 322 & 100 & & 1227 & 100 & & 1850 & 100 & & \\
\hline
\end{tabular}


Table 5 (continued)

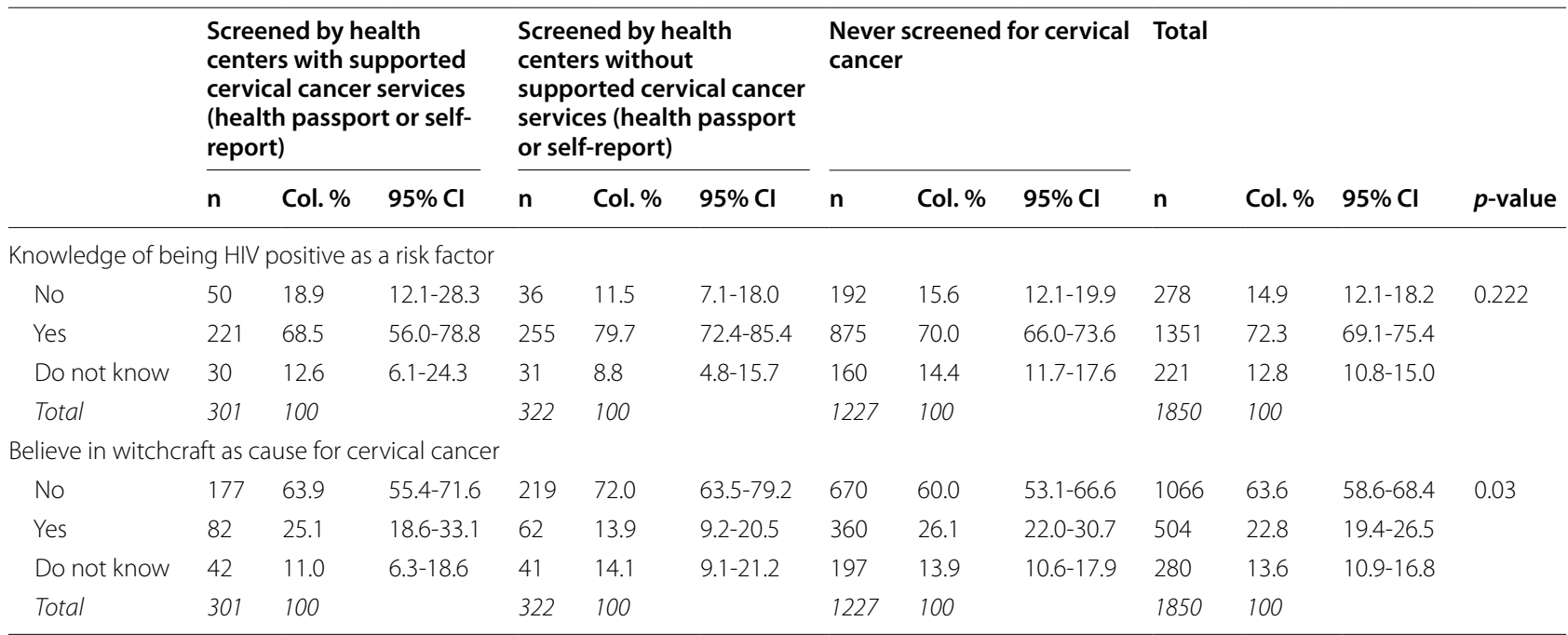

a rather long way to go to reach routine cervical cancer screening among eligible women.

The few surveys that have been published on cervical cancer screening coverage in Malawi and other African countries were in areas with low coverage where no more than a quarter of the survey population had been screened, with the exception of Cameroon where almost half of the survey population had been screened [30, 3741]. Our survey therefore provides an important contribution to this topic.

Knowledge of cervical cancer, risk factors, and possible prevention methods are present in the survey population. However, less than half of the women who have heard of cervical cancer screening have been screened. Likewise, only slightly more than one-third of women who said they believe they are at risk for cervical cancer have been screened. This suggests that knowledge of cervical cancer screening and awareness of being themselves at risk of cervical cancer are not the main barriers to screening in this population. Although the survey participants know that screening is important, and more than half thought they were personally at risk, it did not translate into action. Increasing knowledge about cervical cancer and screening alone is therefore not enough, practical actions that women can do to prevent cervical cancer should be clearly communicated. Information campaigns on cervical cancer risk and prevention possibilities must be reinforced, and specifically target women who have not yet accessed cervical cancer screening services.

These results are consistent with recent qualitative surveys, which showed high awareness but low uptake of cervical cancer screening not only in Malawi [34, 37, 42], and also in other African countries [27, 28, 38, 40,
43-45]. The results are also in line with surveys carried out in Kenya, where higher screening rates were observed in women with higher levels of education in the highest income quintile and living in urban areas $[41,46]$.

Overall, almost all women reported knowledge of cervical cancer, and among those not screened, very few reported fear as a deterrent to screening, or that they would not be comfortable being screened by a man. In Blantyre City, an urban setting with better access to care the main reason for not being screened was lack of time, in Chiradzulu District with access to free and enhanced CC screening services the main reason was lack of information and in Chiradzulu without access to free and enhanced $\mathrm{CC}$ screening services the main reason was inconvenient location. While the predominant reasons for not being screened differed by strata, and can be addressed specifically in each setting, they were consistent in that the barriers were practical, mutable constructs that are amenable to intervention [34, 47]. They included supply-side barriers, (such as lack of time, and lack of access) which can be addressed by the health system, for example by extending hours of service provision, and accessibility of cervical cancer screening sites. In addition, demand-side barriers were important (such as lack of motivation and lack of information), which can be addressed by adapting and scaling up information campaigns. These results are internally consistent with the reasons women reported for screening, which for a majority was due to a recommendation in a health facility, indicating that cervical cancer screening was often an 'add-on' service that women receive when they are already seeking care for other services. The higher proportion of women with HIV among women screened in 
Chiradzulu District is also consistent with the understanding that women undergo cervical cancer screening while they are already in the health facility for other services.

Among all interviewed women who underwent cervical cancer screening in the past, less than $1 \%$ were VIA positive. This is a surprisingly low VIA positivity rate in light of previous studies, including a country wide study reporting 10\% VIA positivity or suspected cancer [25], and a retrospective survey in Kamuzu Central Hospital in Lilongwe, Malawi, reporting almost one-third of HIVpositive women having either high-grade dysplasia or cervical cancer [48]. Considering the $13 \%$ prevalence of HIV in Malawi and the 5\% prevalence of cervical HPV 16 or 18 infections among women in the general population in Eastern Africa [14], a higher proportion VIA positive would be expected. Further research should be envisaged to better understand the real prevalence of VIA positivity and precancer lesions in Malawi.

Although only slightly more than $2 \%$ of women refused to participate in the survey, this represented every ninth women in Blantyre compared to less than 1\% in Chiradzulu. MSF is very well-known in Chiradzulu District, as it has been supporting the health system in the district for many years, whereas MSF's interventions in Blantyre only started with the cervical cancer screening project in 2018. The urban Blantyre population may have had less time and therefore been less willing to participate in the survey, especially as there are many surveys carried out in this city.

Although spatial sampling is an appropriate sample design for urban areas, cluster sampling would have been feasible for Blantyre as the city includes many nonresidential areas, such as arable lands, fields, industrial areas, wasteland and nature reserves; and the population is fairly concentrated in certain areas. As well, the use of geospatial sampling in Blantyre City might have led to over-representation of wealthier women in the survey since they usually live in larger homes with a higher chance to be included in the survey, which may have biased the screening coverage results. However, the distribution of socio-demographic characteristics among participants is similar to the 2015-2016 Malawi Demographic and Health Survey [12], and the differences in socio-demographic characteristics between Blantyre and Chiradzulu rather more likely reflect the differences that usually exist between urban and rural areas than a sampling bias.

\section{Conclusions}

Less than half of eligible women went for cervical cancer screening both in Blantyre City and in Chiradzulu District. These coverage results are slightly higher than previous surveys in Malawi, they are still much lower than the targeted cervical cancer screening rate of $80 \%$ for Malawi.

Most of the survey population had already heard about cervical cancer. Despite this knowledge, fewer than half of eligible women had been screened for cervical cancer. Reasons given for not attending screening are mutable concepts, such as lack of time, access, motivation and information, which are amenable to intervention. Most women who had been screened did so upon recommendation in a health facility, indicating not only that health care workers are successfully promoting screening to women already seeking care, and also that further efforts to inform and motivate women outside of the health facility on practical actions to prevent cervical cancer are necessary. Despite the low cervical cancer screening coverage, the positive reception amongst women who were screened, underscores this core part of cancer prevention as an important part of the way forward to reducing the burden of cervical cancer in Malawi. To significantly reduce mortality due to cervical cancer in Malawi requires a comprehensive health strategy that focuses on prevention, screening and treatment. Cervical cancer is not yet a disease of the past. Cervical cancer is a disease that can be prevented and treated; we know how, we now have to put it in place.

\section{Abbreviations}

ART: Anti-retroviral therapy; CC: Cervical Cancer; EAs: Enumeration Areas Chiradzulu District; HPV: Human papillomavirus; HIV: Human Immunodeficiency Virus; MoH: Malawi Ministry of Health; MSF: Médecins Sans Frontières; VIA: Visual inspection with acetic acid.

\section{Acknowledgements}

We would like to acknowledge the entire MSF team in Malawi and elsewhere for its support and help. Our sincere gratitude goes to the survey team and its supervisors. Many thanks to Sandra Cohuet and Estelle Pasquier for their substantial input in writing the survey protocol. Finally, this survey would not have been possible without the cooperation and generosity of the citizens of Malawi.

\section{Authors' contributions}

SG was the principal study investigator of the survey, being responsible for the conception and design of the survey, supervision of the field data collection, the data analysis and the interpretation of findings. She also drafted the article and approved the final manuscript. LL contributed substantially to the acquisition of data, organised the field data collection, the data analysis, assisted in data interpretation, revised the article critically for important intellectual content and approved the final manuscript. RN, CM and AL contributed substantially to the concept and the design of the survey, assisted in data interpretation, revised the article critically for important intellectual content and approved the final manuscript. TP, JK and HS contributed substantially to the concept of the survey, assisted in data interpretation, revised the article critically for important intellectual content and approved the final manuscript.

Funding

MSF was the survey sponsor and was responsible for the funding.

Availability of data and materials

The data sets generated during the survey are available from the corresponding author upon request. 


\section{Declarations}

\section{Ethics approval and consent to participate}

Ethical approval of the survey was granted by the National Health Science Research Committee in Malawi (approval number: 2377) and MSF ethical review board (approval number: 1944). The survey was carried out in accordance with the in accordance with the relevant guidelines and regulations, the Helsinki Declaration [49] and the applicable national and local laws and regulatory requirements.

This survey was carried out in close collaboration of Epicentre, MSF and the $\mathrm{MoH}$. Several levels of informed agreement and consent were obtained during this survey, including authorities on all administrative and health levels, head of villages and head of households for participation. Written informed consent was provided by the participants. Participants were informed that participation was voluntary, and they could withdraw at any time. Personal identifiers were not included in the questionnaires to ensure participant confidentiality. There was no financial compensation for participating in this survey.

\section{Consent for publication}

Not applicable.

\section{Competing interests}

The authors declare that they have no competing interests.

\section{Author details}

${ }^{1}$ Epicentre / Médecins Sans Frontières (MSF), Paris, France. ${ }^{2}$ Médecins Sans Frontières (MSF), Paris, France. ${ }^{3}$ Médecins Sans Frontières (MSF), Blantyre, Malawi. ${ }^{4}$ Ministry of Health, Blantyre, Malawi.

Received: 12 November 2020 Accepted: 3 January 2022 Published online: 14 February 2022

\section{References}

1. Bray F, Ferlay J, Soerjomataram I, Siegel RL, Torre LA, Jemal A. Global cancer statistics 2018: GLOBOCAN estimates of incidence and mortality worldwide for 36 cancers in 185 countries. CA Cancer J Clin. 2018; 68:394-424.

2. Arbyn M, Weiderpass E, Bruni L, de Sanjosé S, Saraiya M, Ferlay J, Bray F. Estimates of incidence and mortality of cervical cancer in 2018: a worldwide analysis. The Lancet Global Health. 2020;8:e191-e203

3. Knaul FM, Rodriguez NM, Arreola-Ornelas H, Olson JR. Cervical cancer: lessons learned from neglected tropical diseases. Lancet Glob Heal. 2019;7:e299-300.

4. Vaccarella S, Lortet-Tieulent J, RS, Conway DI, Straif K, WC. Reducing social inequalities in cancer: evidence and priorities for research; 2019.

5. Simms KT, Steinberg J, Caruana M, Smith MA, Lew J Bin, Soerjomataram I. Impact of scaled up human papillomavirus vaccination and cervical screening and the potential for global elimination of cervical cancer in 181 countries. Lancet Oncol. 2020:20:394-407.

6. Cubie HA, Campbell C. Cervical cancer screening - the challenges of complete pathways of care in low-income countries: focus on Malawi. Women's. Health. 2020;16:1-10.

7. Singh G. Global inequalities in cervical Cancer incidence and mortality are linked to deprivation, low socioeconomic status, and human development. Int J MCH AIDS. 2012;1:17-30.

8. Ginsburg O, Bray F, Coleman MP, Vanderpuye V, Eniu A, Kotha SR. The global burden of women's cancers: a grand challenge in global health. Lancet. 2017:389:847-60.

9. Gossa W, Fetters MD. How should cervical Cancer prevention be improved in LMICs? AMA J Ethics. 2020;22:E126-34.

10. Ferlay J, Ervik M, Lam F, Colombet M, Mery L, Piñeros M, Znaor A, Soerjomataram I BF. No Title Global Cancer Observatory: Cancer Today 2020 https://gco.iarc.fr/today. Accessed 28 Oct 2021.

11. Bruni L, Albero G, Serrano B, Mena M, Gómez D, Muñoz J, et al. Human Papillomavirus and Related Diseases Report. 2019.

12. National Statistical Office (NSO) [Malawi] and ICF: Malawi Demographic and Health Survey 2015-16. Zomba and Rockville: NSO and ICF; 2017.
13. UNAIDS. UNAIDS Malawi Overview. 2018. [cited 2020 Nov 12]. Available from: https://www.unaids.org/en/regionscountries/countries/malawi.

14. Schiffman M, Castle PE. The promise of global cervical-cancer prevention. N Engl J Med. 2005;353:2101-4

15. Motti PG, Dallabetta GA, Daniel RW, Canner JK. Cervical abnormalities, human papillomavirus, and human immunodeficiency virus infections in women in Malawi. J Infect Dis. 1996:173:714-7.

16. Esber A, Rao N, Norris A, Reese PC, Kandodo J, Nampandeni P, et al. Intravaginal practices and prevalence of sexual and reproductive tract infections among women in rural Malawi. Sex Transm Dis. 2016;43:750-5.

17. Cubie HA, Morton D, Kawonga E, Mautanga M, Mwenitete I, Teakle N. HPV prevalence in women attending cervical screening in rural Malawi using the cartridge-based Xpert ${ }^{\circledR}$ HPV assay. J Clin Virol. 2017;87:1-4.

18. Bateman LB, Blakemore S, Koneru A, Mtesigwa T, McCree R, Lisovicz NF, et al. Barriers and facilitators to cervical Cancer screening, diagnosis, follow-up care and treatment: perspectives of human immunodeficiency virus-positive women and health care practitioners in Tanzania. Oncologist. 2019;24:69-75.

19. Howitt BE, Herfs M, Tomoka T, Kamiza S, Gheit T, Tommasino M, et al. Comprehensive human Papillomavirus genotyping in cervical squamous cell carcinomas and its relevance to cervical Cancer prevention in Malawian women. J Glob Oncol. 2017:3:227-34.

20. Harris TG, Burk RD, Palefsky JM, Massad LS, Bang JY, Anastos K. Incidence of cervical squamous intraepithelial lesions associated with HIV serostatus, CD4 cell counts, and human papillomavirus test results. J Am Med Assoc. 2005;293:1471-6.

21. Denny L, Boa R, Williamson AL, Allan B, Hardie D, Stan R. Human papillomavirus infection and cervical disease in human immunodeficiency virus-1-infected women. Obstet Gynecol. 2008;111:1380-7.

22. Singh DK, Anastos K, Hoover DR, Burk RD, Shi Q, Ngendahayo L. Human Papillomavirus Infection and Cervical Cytology in HIV-Infected and HIVUninfected Rwandan Women. J Infect Dis. 2009;199:1851-61.

23. The Government of Malawi. Health Sector Strategic Plan II-Towards Universal Health Coverage. 2017.

24. The Government of Malawi. Ministry of Health. National cervical cancer control strategy 2016-2020. 2017.

25. Msyamboza KP, Phiri T, Sichali W, Kwenda W, Kachale F. Cervical cancer screening uptake and challenges in Malawi from 2011 To 2015: retrospective cohort study. BMC Public Health. 2016;16:806.

26. Hoque ME, Ghuman S, Coopoosmay R, Van Hal G. Cervical cancer screening among university students in South Africa: a theory based study. PLoS One. 2014:9:1-6.

27. Maree JE, Moitse KA. Exploration of knowledge of cervical cancer and cervical cancer screening amongst HIV-positive women. Curationis. 2014;37:1209.

28. Bukirwa A, Mutyoba JN, Mukasa BN, Karamagi Y, Odiit M, Kawuma E, et al. Motivations and barriers to cervical cancer screening among HIV infected women in HIV care: a qualitative study. BMC Womens Health. 2015;15:111. https://doi.org/10.1186/s12905-015-0243-9.

29. Rosser JI, Njoroge B, Huchko MJ. Knowledge about cervical cancer screening and perception of risk among women attending outpatient clinics in rural Kenya. Int J Gynecol Obstet. 2015;128:211-5.

30. Mabelele MM, Materu J, Ng'ida FD, Mahande MJ. Knowledge towards cervical cancer prevention and screening practices among women who attended reproductive and child health clinic at Magu district hospital, Lake zone Tanzania: a cross-sectional study. BMC Cancer. 2018;18:1-8.

31. Fort VK, Makin MS, Siegler AJ, Ault K, Rochat R. Barriers to cervical cancer screening in Mulanje, Malawi: a qualitative study. Patient Prefer Adherence. 2011;5:125-31.

32. Chadza E, Chirwa E, Maluwa A, Malata A, Kazembe A, Chimwaza A. Factors that contribute to delay in seeking cervical cancer diagnosis and treatment among women in Malawi. Health (Irvine Calif). 2012;04:1015-22.

33. Maseko FC, Chirwa ML, Muula AS. Client satisfaction with cervical cancer screening in Malawi. BMC Health Serv Res. 2014;14:1-8.

34. Moucheraud C, Kawale P, Kafwafwa S, Bastani R, Hoffman RM. "It is big because it's ruining the lives of many people in Malawi": Women's attitudes and beliefs about cervical cancer. Prev Med Rep. 2020;18:101093.

35. Guvenc G, Akyuz A, Açikel CH. Health belief model scale for cervical Cancer and pap smear test: psychometric testing. J Adv Nurs. 2011;67:428-37. 
36. Cancer Research UK, University College London, King's College London and University of Oxford. Cervical Cancer Awareness Measure Toolkit (Cervical CAM), version 2.1. [cited 2021 Nov 23]. Available from: https://doc.ukdat aservice.ac.uk/doc/6646/mrdoc/pdf/6646cervical_cam_toolkit.pdf.

37. Maree JE, Kampinda-Banda M. Knowledge and practices of cervical Cancer and its prevention among Malawian women. J Cancer Educ. 2020;35:86-92.

38. Donatus L, Nina FK, Sama DJ, Nkfusai CN, Bede F, Shirinde J. Assessing the uptake of cervical cancer screening among women aged 25-65 years in Kumbo West Health District, Cameroon. Pan Afr Med J. 2019;33:106.

39. Bante SA, Getie SA, Getu AA, Mulatu K, Fenta SL. Uptake of pre-cervical cancer screening and associated factors among reproductive age women in Debre Markos town, Northwest Ethiopia, 2017. BMC Public Health. 2019;19:1102

40. Gatumo M, Gacheri S, Sayed AR, Scheibe A. Women's knowledge and attitudes related to cervical cancer and cervical cancer screening in Isiolo and Tharaka Nithi counties, Kenya: A cross-sectional study. BMC Cancer. 2018;18:745.

41. Ng'ang'a A, Nyangasi M, Nkonge NG, Gathitu E, Kibachio J, Gichangi P. Predictors of cervical cancer screening among Kenyan women: Results of a nested case-control study in a nationally representative survey. BMC Public Health. 2018;18:1221.

42. Lee H, Mtengezo J, Kim D, Makin M, Kang Y, Malata A. Exploring Complicity of Cervical Cancer Screening in Malawi: The Interplay of Behavioral, Cultural, and Societal Influences. Asia-Pacific J Oncol Nurs. 2019;7:18-27.

43. Ndejjo R, Mukama T, Kiguli J, Musoke D. Knowledge, facilitators and barriers to cervical cancer screening among women in Uganda: a qualitative study. BMJ Open. 2017;7:e016282.

44. Matenge TG, Mash B. Barriers to accessing cervical cancer screening among HIV positive women in Kgatleng district, Botswana: A qualitative study. PLoS One. 2018;13:e0205425

45. Buchanan Lunsford N, Ragan K, Lee Smith J, Saraiya M, Aketch M. Environmental and psychosocial barriers to and benefits of cervical Cancer screening in Kenya. Oncologist. 2017;22:173-81.

46. Kangmennaang J, Onyango EO, Luginaah I, Elliott SJ. The next sub Saharan African epidemic? A case study of the determinants of cervical cancer knowledge and screening in Kenya. Soc Sci Med. 2018;197:203-12.

47. Rahman R, Clark MD, Collins Z, Traore F. Cervical cancer screening decentralized policy adaptation: an African rural-context-specific systematic literature review. Global Health Action. 2019;12: 1587894.

48. Kohler RE, Tang J, Gopal S, Chinula L, Hosseinipour MC, Liomba NG, et al. High rates of cervical cancer among HIV-infected women at a referral hospital in Malawi. Int J STD AIDS. 2016;27:753-60.

49. General Assembly of the World Medical Association. World Medical Association Declaration of Helsinki: ethical principles for medical research involving human subjects. J Am Coll Dent. 2014;81:14-8.

\section{Publisher's Note}

Springer Nature remains neutral with regard to jurisdictional claims in published maps and institutional affiliations.

Ready to submit your research? Choose BMC and benefit from:

- fast, convenient online submission

- thorough peer review by experienced researchers in your field

- rapid publication on acceptance

- support for research data, including large and complex data types

- gold Open Access which fosters wider collaboration and increased citations

- maximum visibility for your research: over $100 \mathrm{M}$ website views per year

At BMC, research is always in progress.

Learn more biomedcentral.com/submissions 